KIRKhaM, D. S. (1957). J. gen. Microbiol. 17, 491-504

\title{
The Significance of Polyphenolic Metabolites of Apple and Pear in the Host Relations of Venturia inaequalis and Venturia pirina
}

\author{
By D. S. KIRKHAM \\ East Malling Research Station, Kent
}

SUMMARY: Series of apple and pear shoots were inoculated with the scab pathogens Venturia inaequalis and $V$. pirina and injected with polyphenolic metabolites extracted from apple and pear leaves, and with urea. The translocation and persistence of arbutin in Cox leaves was demonstrated chromatographically. Injection of metabolites of the highly susceptible varieties Cox and Williams back into the same varieties, markedly inhibited the pathogens, which suggests that these potential resistance factors do not occur only in varieties which are scab resistant. Inhibition of infection was most marked on leaves already developing a mature resistance and the injection treatments were most effective when applied near the time of inoculation. Injection of urea increased susceptibility to $V$. inaequalis, and further results indicated the importance of optimal balance mechanisms between polyphenolic and nitrogenous metabolites. Chlorogenic acid and isochlorogenic acid strongly inhibited both pathogens and there was some indication of synergism between them as resistance factors.

Results obtained in artificial culture (Kirkham, 1957 $b$ ) have shown that polyphenolic host metabolites in relation to other nutritional factors are of potential significance in the scab diseases of apple and pear, caused by Venturia inaequalis (Cke.) Wint. and V. pirina Aderh., respectively. To obtain more direct evidence of the importance of these compounds, injection trials were carried out on series of inoculated apple and pear shoots under glass. Roach (1934) obtained results in which the incidence of mildew (Podosphaera leucotricha) on M. III rootstocks was decreased by injection of a solution of sodium thiosulphate, thus demonstrating the potential value of a plant injection technique in studies of disease reaction. In the present study the Roach method of petiole injection (1938) was used in an attempt to induce variations, both qualitative and quantitative, in the polyphenolic and nitrogenous metabolites within series of similar apple and pear shoots so that effects of these variations on the incidence of the diseases could be observed. Some results of this study have been reported (Kirkham, 1954).

\section{METHODS}

Methods similar to those described in a previous paper (Kirkham, 1957 a) for the determination of host ranges and pathogenicity of distinct clones of Venturia inaequalis and V. pirina were used. The trees to be used for the season's work were moved into the greenhouse serially, as three groups, between the beginning of March and the end of May, to provide material for three 
successive inoculation and injection trials during the spring and early summer. On completion of each trial the four shoots on each tree were cut back to the first well-developed bud and, conditions permitting, these buds produced shoots which were ready for use later in the season. Thus a succession of shoots was maintained from May to October or November. To increase further the length of the period in which trials could be carried out, shoots were raised under artificial light in a heated greenhouse. After their first season of growth, trees were chilled at $5^{\circ}$ during December and then transferred, after pruning, to a small greenhouse heated to a minimal temperature of $13^{\circ}$. Groups of twenty-four trees were illuminated with one $500 \mathrm{~W}$. tungsten filament lamp and two $400 \mathrm{~W}$. mercury vapour lamps to give a minimal day length of $16 \mathrm{hr}$. In these conditions trees of Cox's Orange Pippin and Williams' Bon Chrétien produced visibly normal shoots between 7 and $12 \mathrm{in}$. long by the middle of February.

Monoconidial isolates of each pathogen were used as sources of inoculum. Cultures of Venturia inaequalis, clones E1, A7 and A20 isolated from the varieties Edward VII, Worcester Pearmain and Laxton's Superb respectively, and $V$. pirina, clones $\mathrm{W} 1$ and W3 isolated from Williams' Bon Chrétien, were grown on filter paper-cylinders standing in $10 \%(\mathrm{w} / \mathrm{v})$ malt-extract solution (Kirkham, 1956). The cultures of $V$. inaequalis were used directly for the inoculation of the main trials. Inoculum of $V$. pirina was finally prepared from leaves of Williams after two serial transfers of the pathogen on this variety, because of poor sporulation in culture and loss of pathogenicity during storage.

The immature leaves at the tip of each shoot were inoculated. In the first series of trials (1953) the designation $L_{0}$ was applied to the first fully unrolled leaf below each shoot tip, but in the second series (1954) when the disease reactions of leaves approaching maturity were under investigation, this designation was applied to the second fully unrolled leaf. Leaves below this point were numbered $-1,-2$, etc., and above, $+1,+2$, etc. Leaf $\mathrm{L}_{0}$ in the second series is therefore equivalent to leaf -1 in the first series. Three shoots on each tree were inoculated and the fourth remained as an uninoculated control. Each shoot was enclosed in a polythene-sleeve moist chamber and inoculated with a conidial suspension by using a glass throat atomizer. This method of inoculation was adopted to secure a more even distribution of conidia on the leaves than had been achieved in previous studies (Kirkham, 1957 a). A further improvement was obtained in 1954, when leaves +1 to -1 on apple shoots, and leaves +2 to -2 on pear shoots, were each sprayed separately. The inoculum concentrations were visually adjusted to a turbidity (Spekker drum reading) of c. $0 \cdot 1$ and approximately $1 \mathrm{ml}$. was used for each shoot.

Shoots were injected either before or after inoculation with $1 \%(\mathrm{w} / \mathrm{v})$ aqueous solutions of extracts of the polyphenolic metabolites of Cox's Orange Pippin and Williams' Bon Chrétien (the Cox 3 and Williams 3 extracts described by Kirkham, $1957 b$ ), pure components of these extracts, or urea. Preliminary attempts were made to assess effects of these components and 
mixtures on the incidence of the diseases after injection of inoculated shoots through single petioles (leaf -2 ) but no significant differences were observed between disease reactions on permeated and non-permeated leaf zones, owing to the uneven distribution of inoculum. It was therefore necessary to induce complete permeation of each shoot tip, and to compare disease incidence on a series of shoots. With an aqueous solution of acid fuchsin $(1 \%, w / v)$, it was found that complete permeation resulted from injection through three petioles. Thus, in the trials described below, leaves $-2,-3$ and -4 were used on apple shoots and $-3,-4$ and -5 on pear shoots. The laminae were removed from the leaves and the petioles dipped into the injectant contained in polythene tubes wired on to the shoots. Nine shoots on three trees were used in each injection treatment. The solutions were Seitz-filtered before use to avoid blockage of the vessels by solid particles during injection. Between 3 and $5 \mathrm{ml}$. of injectant were usually taken in by each shoot, depending on the nature and concentration of the solution and the external conditions during injection. The following conditions were necessary for maximal intake:

(a) A greenhouse temperature of $18-24^{\circ}$. Below $18^{\circ}$ intake was very slow and above $24^{\circ}$ too rapid, the latter resulting in wilting and damage to the shoots. (b) Moderate air turbulence to increase transpiration; this was achieved by the use of an electric fan. (c) A light intensity sufficiently high to maintain active photosynthesis. During 1953 much difficulty was experienced in producing these conditions at the required times and therefore, during the following year, two $500 \mathrm{~W}$. tungsten and four $400 \mathrm{~W}$. mercury vapour lamps were used to provide illumination during injection.

Disease incidence, on a series of shoots to which various injection treatments had been applied, was observed by visual estimation of lesion development at frequent intervals after the first appearance of lesions. Both surfaces of each inoculated leaf were recorded separately by estimation of $\%$ surface area covered by sporulating lesions. Leaves $-1, \mathrm{~L}_{0}$ and +1 were recorded on apple shoots and leaves -2 to +2 on pear shoots as more leaves usually became diseased on the latter. In each treatment nine replicate leaves (eighteen surfaces) were recorded in each leaf category, and the average for the category was described as the mean percentage lesion cover. Observations were also made on fleck reactions and on leaf damage due to various causes. The results are illustrated graphically, relating mean percentage lesion cover to the time (in days) after inoculation, for separate leaf numbers. The relationship between the probit values of the mean percentages and the logarithms of the times appeared to be approximately linear and so probit and logarithm scales were used for convenience. With one exception (trial 3/54), large differences resulted from the various treatments, and a high degree of correlation was observed between the results of similar trials carried out on separate occasions. In these circumstances the results, excepting those of trial 3/54, were not analysed statistically.

The chromatograms were run as previously described (Kirkham, 1957b). 


\section{RESULTS}

Inhibition of Venturia inaequalis and $\mathrm{V}$. pirina on leaves injected with polyphenolic host metabolites

Cox trees on M. IV rootstocks were inoculated with Venturia inaequalis, clone E 1. Twenty-four hr. later nine shoots were injected with the Cox extract (Cox injection 1), nine shoots with water, and eighteen were left uninjected. Twelve days after inoculation, when lesion traces were visible, nine of the uninjected shoots were treated with the same Cox injectant (Cox injection 2). The volume taken up by each shoot was approximately $2.5 \mathrm{ml}$. Symptoms of iron deficiency were visible in the material and the trees were arranged, as far as possible, to include all stages of severity in each treatment. No damage resulted from the injections, but some leaves were lost by sun-scald during inadequate shading of the greenhouse. Fig. 1 illustrates the incidence of disease recorded on -1 leaves in this trial (no. 4/53). The first Cox injection markedly reduced lesion development but the second, 12 days after inoculation, was less effective. Leaves in the $\mathrm{L}_{0}$ category showed similar disease reactions but there was less inhibition and less effect of time of injection. No inhibition was observed in the water treatment.

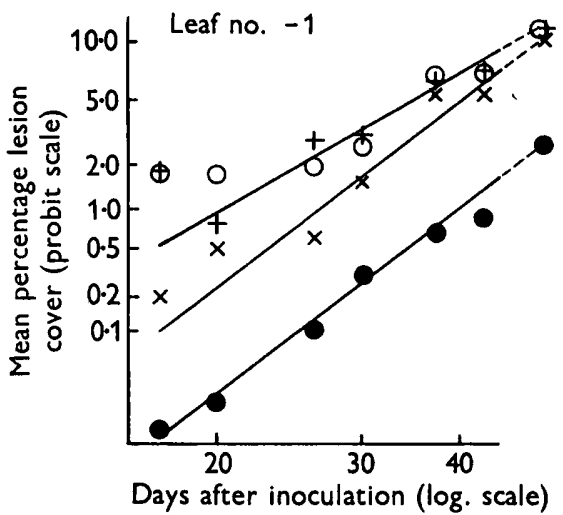

Fig. 1. Inhibition of Venturia inaequalis, clone $\mathrm{E} 1$ on $\mathrm{Cox}$, by injection of polyphenolic metabolites of Cox (Trial 4/53). Cox 1 : injected $24 \mathrm{hr}$. after inoculation; Cox 2: injected 12 days after inoculation. Injections: + , no injection; $O$, water; $O, \operatorname{Cox} 1 ; \times, \operatorname{Cox} 2$.

Trial 10/53 showed similar reactions of Venturia pirina (clone W1) on Williams' pear. Twenty-four hr. before inoculation nine shoots were injected with the Williams' extract and nine shoots with water. The intake of each injectant was approximately $3 \mathrm{ml}$./shoot and no leaf damage was observed. Figs. 2-4 show the reactions observed on $-2,-1$ and $\mathrm{L}_{0}$ leaves respectively. Considerable inhibition of the pathogen occurred on -2 leaves but on $L_{0}$ leaves where the mean \% lesion cover on the controls was considerably higher, only very slight inhibition resulted from the injection of host metabolites. Disease incidence on -1 leaves was intermediate between reactions on $\mathbf{- 2}$ 
and $\mathrm{L}_{0}$ leaves. These results confirmed indications from small-scale trials on trees raised under artificial light, where inhibition of Venturia inaequalis, clones E1 and A26 on Cox and V. pirina, clones W1 and P16 on Williams had been observed.

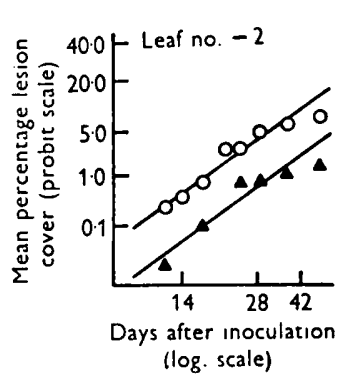

Fig. 2

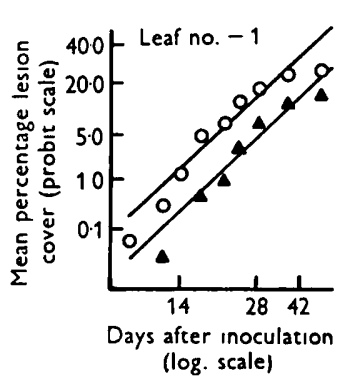

Fig. 3

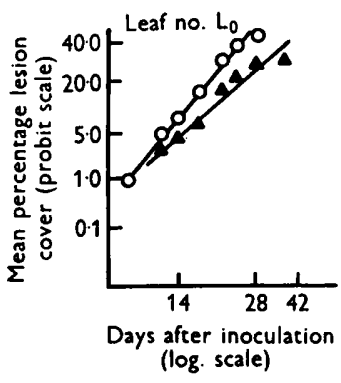

Fig. 4

Figs. 2-4. Inhibition of Venturia pirina, clone W1 on Williams' pear, by injection of polyphenolic metabolites of Williams. (Trial 10/53.) Leaves $-2,-1$ and $L_{0}$ in decreasing order of age at time of inoculation. Injections: $O$, water; $\boldsymbol{\Delta}$, Williams.

The question arises as to whether or not these reactions resulted from the actual presence of injected compounds in the leaves. The situation is unusual in that compounds already present were injected, and it therefore seems reasonable to suppose that translocation might occur, and that concentration increases might be tolerated to some extent. Very rapid host reactions to the injection treatments were sometimes observed, including interveinal desiccation of mature leaves below the injection points. A temporary return to the juvenile pink colour in leaves of Durondeau pear after injection of polyphenolic metabolites was also noted. These observations, however, do not necessarily indicate that translocation occurred unless they can be supported by direct chemical evidence. To obtain this evidence apple seedlings were injected with the pear glycoside arbutin. Twenty-four hr. later, leaves above the injection points were removed and their polyphenolic fraction compared chromatographically with that of uninjected leaves; it was found that the foreign glycoside was present in the injected leaves. It will therefore be assumed, pending further studies of translocation and persistence, that the observed effects on disease incidence resulted from at least the temporary presence of injected compounds in the leaves. Persistence for long periods seems to be unlikely, as the metabolic processes of a leaf would tend to remove foreign compounds, or restore to normal any altered balances between metabolites.

Results obtained in artificial culture (Kirkham, 1957 b) showed that polyphenolic host metabolites may play an important part in the nutrition of the scab pathogens and the trials described above indicate the possible significance of these compounds in host resistance. The incidence of the diseases on Cox apple and Williams' pear was considerably decreased by injection of polyphenolic metabolites which are already present in the highly susceptible leaves. 
It seems that these decreases can be most easily induced on leaves which are already developing a mature resistance and, though this effect may be partially due to concentration of injected compounds above the injection points, later results (see below) showed that large differences in disease reaction could also be obtained on leaves above $\mathrm{L}_{0}$.

\section{Further reactions of Venturia inaequalis to injections of polyphenolic metabolites of Cox's Orange Pippin}

In trial 6/53, injection towards the end of the incubation period of the disease did not affect lesion development on any leaf. Trees of Cox and Worcester on M. II rootstocks were inoculated with Venturia inaequalis, clone $\mathbf{E} 1$ and, 12 days later, injected with Cox 3 extract. Fig. 5 shows that there was no inhibition on -1 leaves of Cox, and similar results were obtained with all other leaves in this trial, irrespective of age and degree of lesion cover on the controls. These results confirm those of trial $4 / 53$, where injection 12 days after inoculation was less effective than injection $24 \mathrm{hr}$. after inoculation. It seems that the pathogen is most susceptible to inhibition during the very early stage of the incubation period of the disease, after penetration of the leaf cuticle and when the development of the subcuticular mycelium is beginning. Similarly, final results in culture were often partially determined by reactions which occurred during the lag phases.
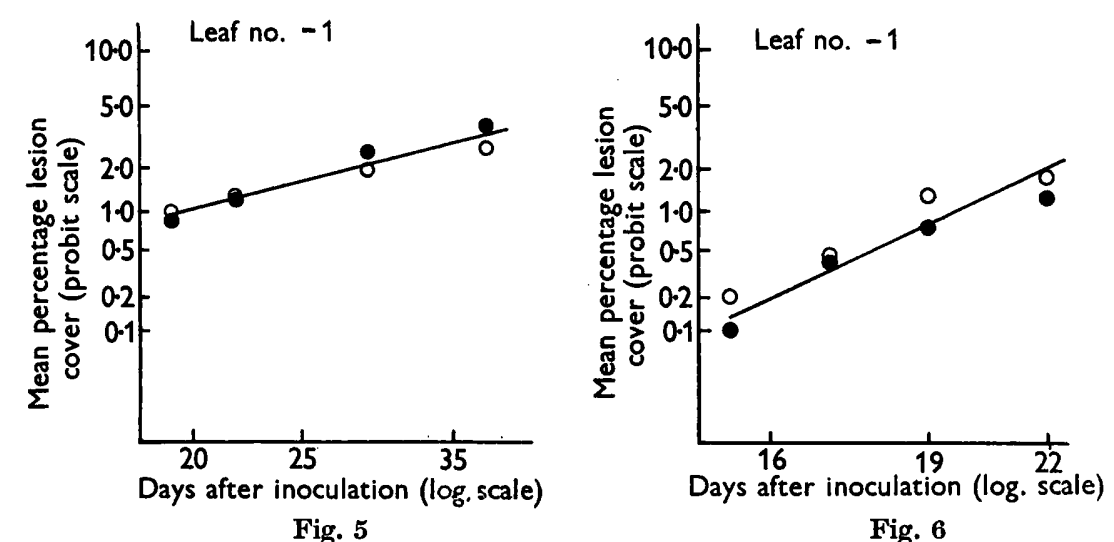

Figs. 5-6. Lack of inhibition of Venturia inaequalis, clone $\mathrm{E} 1$ on Cox and clone A20 on Laxton's Superb, by injection of polyphenolic metabolites of Cox 12 days after and 2 days before inoculation respectively. (Trials 6/53 and 9/53.) Injections: $O$, water; , Cox.

Trial 9/53 also yielded results in which disease incidence was not influenced by Cox 3 extract injection. Trees of Laxton's Superb were inoculated with Venturia inaequalis clone A20, originally isolated from this variety, $24 \mathrm{hr}$. after injection. A high injection intake of approximately $4.5 \mathrm{ml}$./shoot was observed, but there was no inhibition even on -1 leaves (Fig. 6). In the same trial (9/53) Worcester trees were inoculated with clone E1, 24 hr. after 
injection. The intake of each injectant was again approximately $4.5 \mathrm{ml}$./shoot. In contrast to the reaction of clone A 20 on Laxton's Superb, striking inhibition of clone $\mathrm{E} 1$ was observed (Figs. 7, 8). The mean $\%$ lesion cover of the $\mathbf{L}_{\mathbf{0}}$ control leaves was higher than that of the -1 control leaves and as expected, less inhibition was observed on $\mathrm{L}_{0}$ leaves.

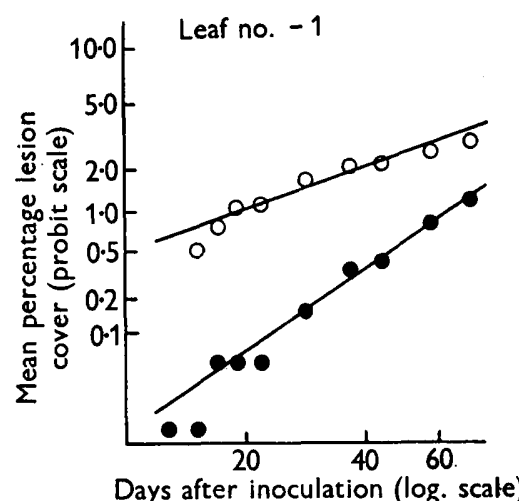

Fig. 7

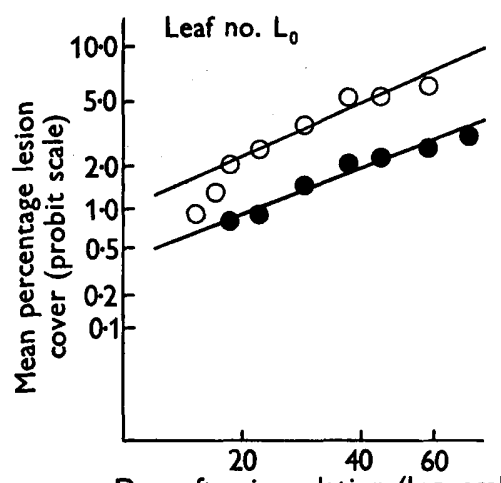

Days after inoculation (log. scale)

Fig. 8

Figs. 7-8. Inhibition of Venturia inaequalis, clone $\mathbf{E} 1$ on Worcester Pearmain, by injection of Cox metabolites $24 \mathrm{hr}$. before inoculation (trial 9/53). Injections : $O$, water; $\bullet$, Cox.

It is clear that injection of polyphenols near the time of inoculation does not necessarily cause inhibition of the pathogen. The occurrence and degree of inhibition will presumably be determined by the particular association of clone and host variety, and does not depend solely on the time of injection. In the case of clone A 20 on Laxton's Superb any quantitative changes induced by injection were insufficient to influence the development of the pathogen. In the same trial, however, the growth of clone $\mathbf{E} 1$ on Worcester was strongly influenced by the injection of Cox metabolites, which again suggests that metabolites contributing to the mechanism of disease resistance can be obtained from highly susceptible as well as less susceptible varieties.

In a previous paper, relationships were demonstrated between the host ranges of distinct clones of Venturia inaequalis and their susceptibility to inhibition by the Cox extract, in artificial culture (Kirkham, 1957b). Clone E1 had the widest host range and was least susceptible, while the converse was found for clone A7, and clone A20 held an intermediate position. In trial $8 \mathrm{a} / 53$ reactions of clones $\mathrm{A} 20$ and $\mathrm{A} 7$ on Cox leaves to injection of the shoots with Cox 3 extract, were observed. Shoots of Cox on M.IV were inoculated, and injected twice with Cox 3 extract during the incubation period of disease, to introduce the maximum volume of injectant into each shoot tip. Twenty-four hr. after inoculation the intake was approximately $\mathbf{3 . 3} \mathrm{ml}$./shoot, and 6 days after inoculation, $5 \mathrm{ml}$./shoot. Owing to lack of sun there was an enforced gap of 5 days between the two injections. Symptoms of iron deficiency, which appeared to be increased by the Cox 3 extract injection, were noted in the material and a complete range of severity was included in each treatment. 
Figs. 9 and 10 show reactions of clones A20 and A7 respectively on -1 leaves. Lesion development of both clones was inhibited and, in contrast to the in vitro results clone A20 was more susceptible than was clone A7. The high injection intake in this trial did not result in increased inhibition of the pathogen, possibly owing to the fact that the second injection treatment was applied too long after the initiation of the disease. If, on the other hand, the second injection was effective, clones A20 and A7 were less susceptible to inhibition in trial $8 \mathrm{a} / 53$ than was clone $\mathrm{E} 1$ in trial 4/53, on Cox. There appeared to be no relationship between the host range of a clone and its reaction to injection of a highly susceptible variety with Cox 3 extract.

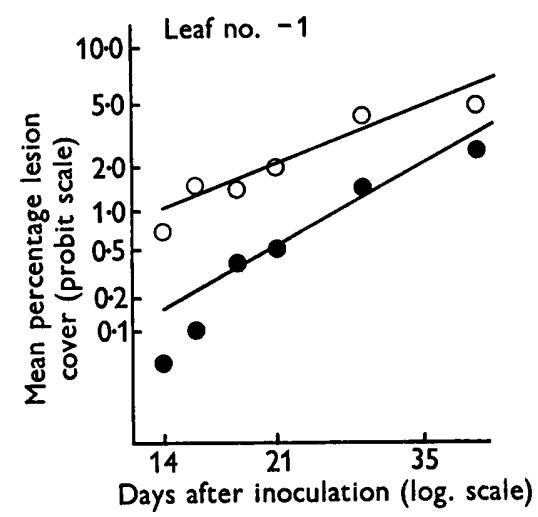

Fig. 9

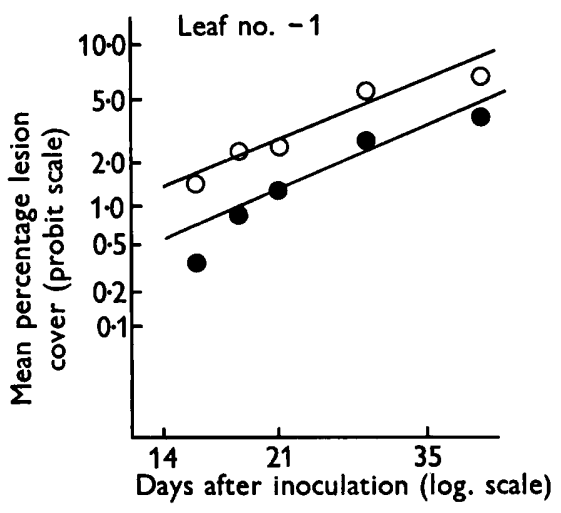

Fig. 10

Figs. 9-10. Inhibition of Venturia inaequalis, clones A 20 and A 7 on Cox, by two injections of Cox metabolites during the incubation period of the disease (trial 8a/53). Injections : O, water; 0 , Cox.

\section{Effects of urea and polyphenolic metabolites on the incidence of Venturia inaequalis on Cox}

Trees of Cox on M. II rootstocks were inoculated with Venturia inaequalis clone $\mathbf{E} 1$, and 5 days later shoots were injected with water, Cox 3 extract, a $0.5 \%(\mathrm{w} / \mathrm{v})$ solution of urea, or a solution containing $1 \%(\mathrm{w} / \mathrm{v}) \operatorname{Cox} 3$ extract $+0.5 \%(\mathrm{w} / \mathrm{v})$ urea. The intake of each injectant was approximately $3.3 \mathrm{ml} . / \mathrm{shoot}$. Injection of urea resulted in marginal scorch which developed slowly on young leaves above the injection points, and during intake of Cox 3 extract rapid interveinal desiccation of leaves below the -2 stage was observed. Pronounced interveinal scorch was visible on these Cox 3 extract treated leaves $12 \mathrm{hr}$. after injection. The mixture induced slight symptoms of damage by urea and Cox 3 extract but was considerably less phytotoxic. Figs. 11 and 12 show disease incidence on -1 and $L_{0}$ leaves respectively (trial $8 \mathrm{~b} / 53$ ). Cox 3 extract markedly reduced lesion development on -1 leaves and urea had the opposite effect. In the latter treatment many diffuse lesions were present on the undersurfaces of the leaves. The mixture was intermediate in activity between urea and the water control but, 28 days after inoculation the mean $\%$ lesion areas in the mixture and water injection 
treatments were the same. Disease incidence was not higher on $\mathrm{L}_{0}$ leaves in this trial, but the Cox 3 extract treatment was, as usual, less effective on these leaves. The urea and mixture injections equally stimulated lesion development on the younger leaves.

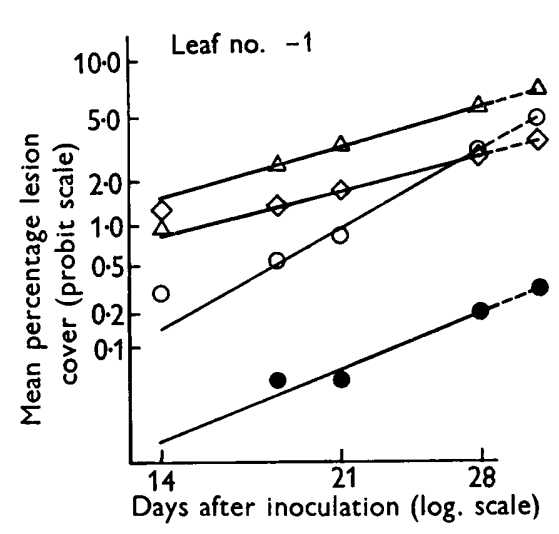

Fig. 11

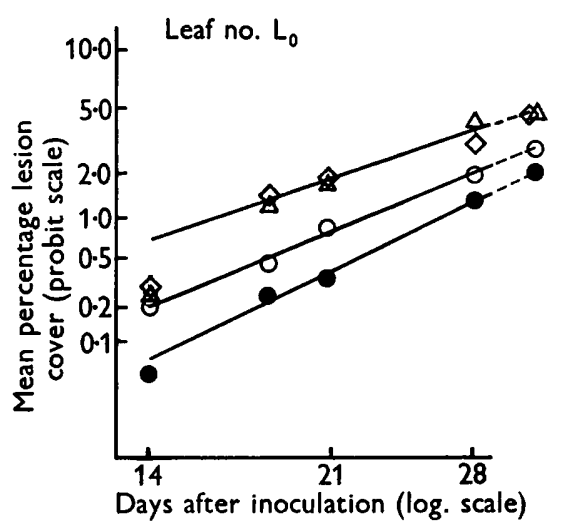

Fig. 12

Figs. 11-12. Inhibition and stimulation of Venturia inaequalis, clone $\mathbf{E} 1$ on Cox, by injection of Cox metabolites, urea and a mixture of these two injectants, 5 days after inoculation (trial 8b/53). Injections: $O$, water; $\diamond$, Cox and urea; $\bullet, \operatorname{Cox;} \Delta$, urea.

Previous results obtained in artificial culture (Kirkham, 1957b) showed that the activity of the polyphenols was influenced by the composition of the basal medium; inhibition was least marked in the presence of an optimal nutritional substrate. Injection of urea would rapidly result in an enrichment of the nitrogenous constituents of the in vivo substrate and might therefore, as suggested by this trial, decrease the inhibition of the pathogen by the naturally occurring polyphenols. In trial $8 \mathrm{~b} / 53$ inhibition by $\operatorname{Cox} 3$ extract was certainly decreased by the injection of urea. There was some indication that the separate effects of the extract and urea might exactly counteract each other, depending on the proportions in the mixture and the age of the leaf. As indicated by the $\mathrm{L}_{0}$ result, less urea would be required to achieve this in younger leaves which naturally contain more nitrogen.

Figs. 13-15 illustrate the results of trial 3/54 in which the same injectants as in trial $8 \mathrm{~b} / 53$ and a $1 \%(\mathrm{w} / \mathrm{v})$ solution of arbutin were used. Trees of Cox on M. IV rootstocks were inoculated with Venturia inaequalis clone E1 2 days before injection. The intake of arbutin was approximately $3 \mathrm{ml}$./shoot, and of the other injectants approximately $4 \mathrm{ml}$./shoot. Urea again caused some marginal scorch on the young leaves and the mixture of Cox 3 extract and urea was less damaging but no interveinal desiccation occurred during injection of $\operatorname{Cox} 3$ extract alone. The rate of intake of arbutin, the main pear glycoside, was slow compared with the other injectants and there was no indication of phytotoxicity. In contrast to all previous results, injection of Cox 3 extract increased the incidence of disease on leaves -1 to +1 . (Leaf $L_{0}$ in the 1954 series of trials is equivalent to leaf -1 in the 1953 series.) It was also noted 
that the mean \% lesion areas on the water-injected shoots in this trial were considerably higher than those resulting from previous combinations of clone E1 and Cox. As relatively small differences between the results of treatments were recorded in trial 3/54, the separate lines on each graph indicate results, or groups of results which differed significantly at the $1 \%$ level. There was no significant interaction ( $5 \%$ level) between days after inoculation and treatments. Cox 3 extract stimulated lesion development equally on all leaves, irrespective of their ages and the disease incidence on the controls. Urea was most stimulatory to the pathogen in leaves approaching maturity and its activity was similar to that of Cox 3 extract at the -1 stage, while the activity of the mixture was similar to that of urea but somewhat greater. There was some indication that the mixture was more active than either Cox 3 extract or urea alone in -1 leaves, and it was less active than Cox 3 extract alone in +1 leaves. Arbutin was inactive, inhibitory or stimulatory, depending on leaf age.

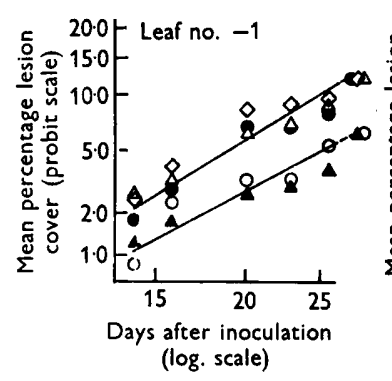

Fig. 13

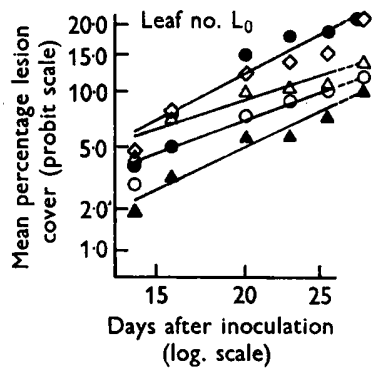

Fig. 14

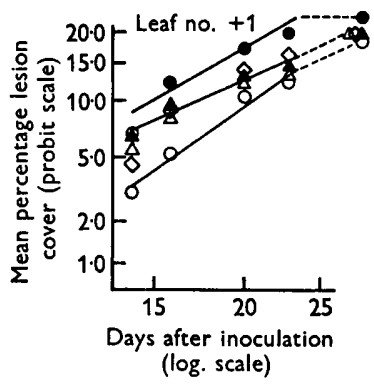

Fig. 15

Figs. 13-15. Stimulation of Venturia inaequalis, clone $\mathbf{E} 1$ on Cox, by injection of Cox metabolites, urea, and a mixture of these two injectants, 2 days after inoculation, and inhibition and stimulation by arbutin (trial 3/54). Injections: $O$, water; 0 , Cox; $\triangle$, urea; $\diamond$, Cox and urea; $\boldsymbol{\Delta}$, arbutin.

The results of trial $8 \mathrm{~b} / 53$ suggested a fairly simple situation in which the reaction of a clone of Venturia inaequalis to the ratio between the polyphenolic and nitrogenous metabolites of an apple variety might be an important factor in the determination of pathogenicity. Trial 3/54, however, showed that the polyphenols may stimulate as well as inhibit the pathogen in vivo. Similar results have been obtained with $V$. pirina in vitro, but stimulation of $V$. inaequalis has not yet been recorded in the artificial environment. The shoots used in trial 3/54 were growing very rapidly at the time of inoculation, and therefore -1 and $L_{0}$ leaves may have been similar to +1 leaves in physiological age and all highly susceptible to the disease, even though $\mathrm{L}_{0}$ leaves were equivalent to -1 leaves in trial $8 \mathrm{~b} / 53$. The injection of Cox metabolites resulting in increased polyphenolic content, perhaps up to a critical concentration above which inhibition would occur, seems to have increased the incidence of disease. A quantitative increase in the nitrogenous metabolites, also up to a critical concentration, might increase the stimulatory effect of the naturally 
occurring polyphenols. This effect would, as observed, be less marked on younger leaves which naturally contain more nitrogen. The mixture of Cox 3 extract and urea was also less active in younger leaves, which suggests that an adverse balance between polyphenolic and nitrogenous metabolites was again established, compared with the balance resulting from injection of Cox 3 extract alone; stimulation was thus reduced. In conditions where Cox 3 extract increased disease incidence on $\mathrm{L}_{0}$ leaves, arbutin had the opposite effect. Although arbutin is not a natural metabolite of the apple, this fact suggests the potential importance of qualitative differences between the polyphenolic metabolites of varieties and leaves of different ages which are characterized by different degrees of resistance.

Effects of polyphenolic metabolites of Williams' pear, including chlorogenic acid and isochlorogenic acid, on Venturia inaequalis and $\mathrm{V}$. pirina on apple and pear shoots, respectively

Trial 1/54 was carried out during February on shoots of Cox on M. IV rootstocks, grown under artificial light in the heated greenhouse. Two days after inoculation with Venturia inaequalis clone $\mathbf{E} 1$, three injection treatments were applied. A $1 \%(\mathrm{w} / \mathrm{v})$ solution of Williams 3 extract and $\mathbf{0} \cdot \mathbf{2} \%(\mathrm{w} / \mathrm{v})$ solution of chlorogenic acid were used in addition to the water control and approximately $4 \mathrm{ml}$. were taken in by each shoot. The rate of intake of Williams 3 extract was slow and variable and leaves above the injection points soon began to show symptoms of phytotoxicity; the midribs became arched, the laminae crinkled and the margins desiccated. Three days after injection the marginal desiccation was visible as blackened areas similar to scorch symptoms on pear leaves. Severely damaged leaves became stunted but shoot extension continued with the production of normal leaves. Intake of chlorogenic acid was rapid, and resulted in similar though much less severe damage. Pl. 1 shows leaves from this trial injected with water, chlorogenic acid, and Williams 3 extract, respectively. The phytotoxic action of the Williams extract seemed to be at least partly due to the chlorogenic acid component, even though this depside is naturally present at a low concentration in Cox leaves. This reaction to injection of Williams 3 extract is in striking contrast to the tolerance shown towards Cox 3 extract, and the pear glycoside arbutin (see above). Fig. 16 shows the results of this trial, which again suggest that chlorogenic acid is a highly active metabolite. Owing to decrease of leaf area, disease incidence in the Williams-extract treatment was unavoidably rated somewhat too highly, and is therefore not exactly comparable with the other treatments. Similar results were observed on $\mathrm{L}_{0}$ leaves.

Some effects of the depsides chlorogenic acid and isochlorogenic acid were observed in trial 2/54. Shoots of Cox on M. IV rootstocks were grown under artificial light and the trees were transferred to an unheated house before inoculation. Two days after inoculation with Venturia inaequalis clone E1, four injection treatments were applied. These included, apart from the water control, solutions of the depsides $(0 \cdot 2 \%, \mathrm{w} / \mathrm{v})$ and another solution containing $0.1 \%$ of each compound. Owing to the insolubility of pure isochlorogenic acid 
in water, it was dissolved in a minimal volume of absolute ethanol before addition of water; all the injectants including the water control therefore contained $1 \%(\mathrm{w} / \mathrm{v})$ ethanol. The intake of each injectant, except the depside mixture, was approximately $4 \mathrm{ml}$./shoot. The depside mixture was injected

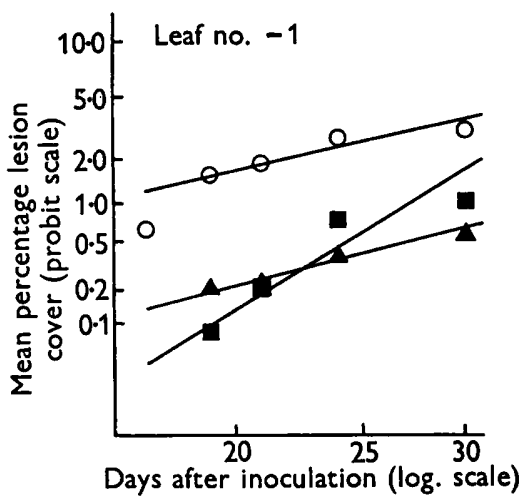

Fig. 16. Inhibition of Venturia inaequalis, clone $\mathrm{E} 1$ on Cox, by injection of polyphenolic metabolites of Williams' pear and chlorogenic acid alone, 2 days after inoculation (trial 1/54). Injections: $O$, water; $\Delta$, Williams; $\mathbf{\square}$, chlorogenic acid.

during cool evening conditions and only $c .3 \mathrm{ml}$. of the solution were taken up by each shoot. Damage following chlorogenic acid injection was again observed, including in this trial some slight interveinal scorch. isoChlorogenic acid was less phytotoxic than chlorogenic acid but induced similar symptoms on -1 leaves. Younger leaves in this treatment slowly developed a slight marginal scorch and a persistent interveinal dark red coloration. Very slight symptoms of chlorogenic acid damage were visible on the mixture-treated shoots, and there was no host reaction to the control injection. Figs. 17-19 show that the depsides inhibited lesion development on leaves -1 to +1 and that their relative activities varied markedly with leaf age. The mixture of chlorogenic acid and isochlorogenic acid was inactive at the -1 stage of leaf

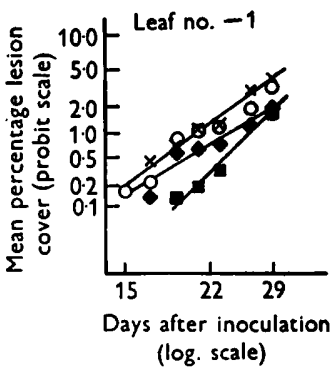

Fig. 17

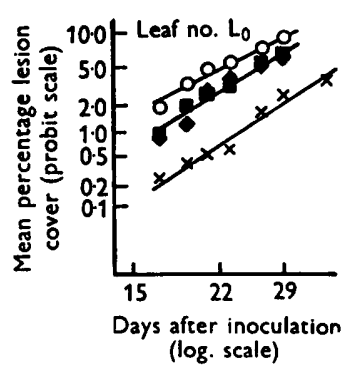

Fig. 18

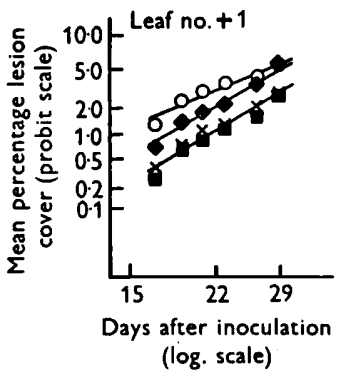

Fig. 19

Figs. 17-19. Inhibition of Venturia inaequalis, clone $\mathbf{E} 1$ on $\mathrm{Cox}$, by injection of chlorogenic acid and isochlorogenic acid as separate and combined injectants, 2 days after inoculation (trial 2/54). Injections: $\bigcirc$, water; $\square$, chlorogenic acid; $\diamond$, isochlorogenic acid; $\times$, chlorogenic acid and isochlorogenic acid. 
development, strongly inhibitory at the $\mathrm{L}_{0}$ stage and equal in activity to chlorogenic acid at the +1 stage.

Reactions of Venturia pirina, clone W3, on shoots of Williams' pear injected with chlorogenic acid or $i$ sochlorogenic acid were observed in trial 4/54 (Fig. 20). Three days after inoculation the same injection treatments were applied as in trial 2/54, but the mixture of chlorogenic acid and isochlorogenic acid was omitted. The intake of each injectant was approximately $3 \mathrm{ml}$. $/ \mathrm{shoot}$ and there was no leaf damage.

As expected, lesion development on the control shoots varied markedly with leaf age, +1 leaves being most susceptible to the pathogen. Chlorogenic acid was inactive at the +1 and $\mathrm{L}_{0}$ stages, and slightly inhibited the early development of the pathogen on -1 leaves. isoChlorogenic acid, however, inhibited clone $\mathbf{W} 3$ at all leaf stages but was least active in +1 leaves. Thus, in contrast to the reactions of Venturia inaequalis on Cox shoots (trial 2/54), isochlorogenic acid was more active against $V$. pirina clone W3 than was chlorogenic acid.

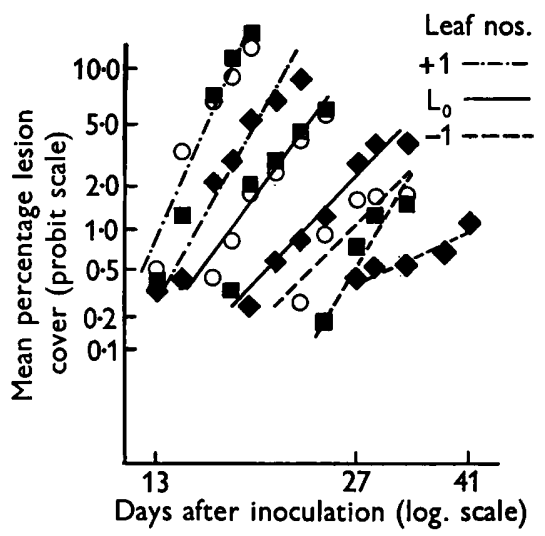

Fig. 20. Inhibition of Venturia pirina, clone W3 on Williams, by injection of chlorogenic acid and $i$ sochlorogenic acid, 3 days after inoculation (trial 4/54). Injections : $O$, water; $\square$, chlorogenic acid; $\diamond$, isochlorogenic acid.

The results of these three trials indicate that the depsides may be important factors in the host relations of Venturia species and that synergism may occur between them as resistance factors. It is thought that trial $\mathbf{4 / 5 4}$ illustrates the results of alterations in the natural chlorogenic acid: isochlorogenic acid ratios in the leaves of Williams' pear and that this ratio may be a significant factor in disease resistance.

\section{DISCUSSION}

The results confirm and extend the hypothesis that polyphenolic host metabolites may be important factors in scab resistance. There are further indications of optimal balance requirements, both within and between the polyphenolic and nitrogenous metabolites, for the establishment of a progressive relationship between host and pathogen. The polyphenols may act as limiting factors in nutrition as suggested by the gradations in response, from stimu- 
lation to inhibition, which have been observed both in the present studies and in artificial culture. The stimulation of Venturia inaequalis resulting from the urea injections supports the conclusions of Johnstone (1931) and of Winkelmann, Holz \& Jaenichen (1937) that a high nitrogen concentration increased susceptibility to apple scab. It is well known that mature leaves of all varieties are immune to infection and that this immunity cannot be explained by the thickness of the cuticular barrier alone. Fleck reactions indicating failure of the pathogens after successful infections were more commonly seen on leaves approaching maturity than on young leaves, and the injection treatments were often more effective on the former. This seems to indicate that, in leaves developing a natural mature resistance, it is easier to induce a balance between metabolites which is less favourable to the pathogen. There appears to be a considerable measure of agreement between the results obtained in artificial culture (Kirkham, 1957b) and in the natural environments of the pathogens. It seems, therefore, that valuable clues to the mechanisms of scab resistance could be obtained from further studies of the pathogens in pure culture.

The author is indebted to Dr R. V. Harris for advice and interest, to Dr W. A. Roach for placing at his disposal a very wide knowledge of plant injection methods, to Dr A. E. Flood for his continued help, particularly in running the chromatograms, to Mr M. M. MacNeill for raising trees under artificial light, to Miss Jean Richardson for technical assistance and to Dr S. C. Pearce for the statistical analysis.

\section{REFERENCES}

Johnstone, K. H. (1931). Observations on the varietal resistance of the apple to scab (Venturia inaequalis Aderh.) with special reference to its physiological aspects. Part II. J. Pomol. 9, 195.

Kirkham, D. S. (1954). Significance of the ratio between the water-soluble aromatic and nitrogen constituents of apple and pear in the host-parasite relationships of Venturia species. Nature, Lond. 173, 690.

KIrKhaM, D. S. (1956). A culture technique for Venturia spp. and a turbidimetric method for the estimation of comparative sporulation. Nature, Lond. 178, 550.

KirkнAM, D. S. (1957a). Relationships between cultural characters and pathogenicity in Venturia inaequalis and Venturia pirina. J. gen. Microbiol. 16, 360.

KirkHaM, D. S. (1957b). Studies of the significance of polyphenolic host metabolites in the nutrition of Venturia inaequalis and Venturia pirina. J. gen. Microbiol. 17, 120.

RoAch, W. A. (1934). Effect of sodium thiosulphate on apple mildew. Ann. appl. Biol. 21, 341.

Roach, W. A. (1938). Plant injection for diagnostic and curative purposes. Tech. Commun. Bur. Hort., E. Malling no. 10.

Winkelmann, A., Holz, W. O. \& Jaenichen, H. (1937). Beiträge zur Biologie und Bekämpfung des Apfelschorfes (Fusicladium dendriticum (Wallr.) Fckl.). III. Mitt. Zbl. Bakt. (2 Abt.), 96, 177.

\section{EXPLANATION OF PLATE}

Fig. 1. Damage to leaves of Cox's Orange Pippin caused by injection of chlorogenic acid and polyphenolic metabolites of Williams' pear $\left(\times \frac{1}{3}\right)$. Left to right: leaves $-1, L_{0}$ and +1 . Upper: water control; middle: chlorogenic acid; lower: Williams 3 extract. 
Journal of General Microbiology, Vol. 17, No. 2

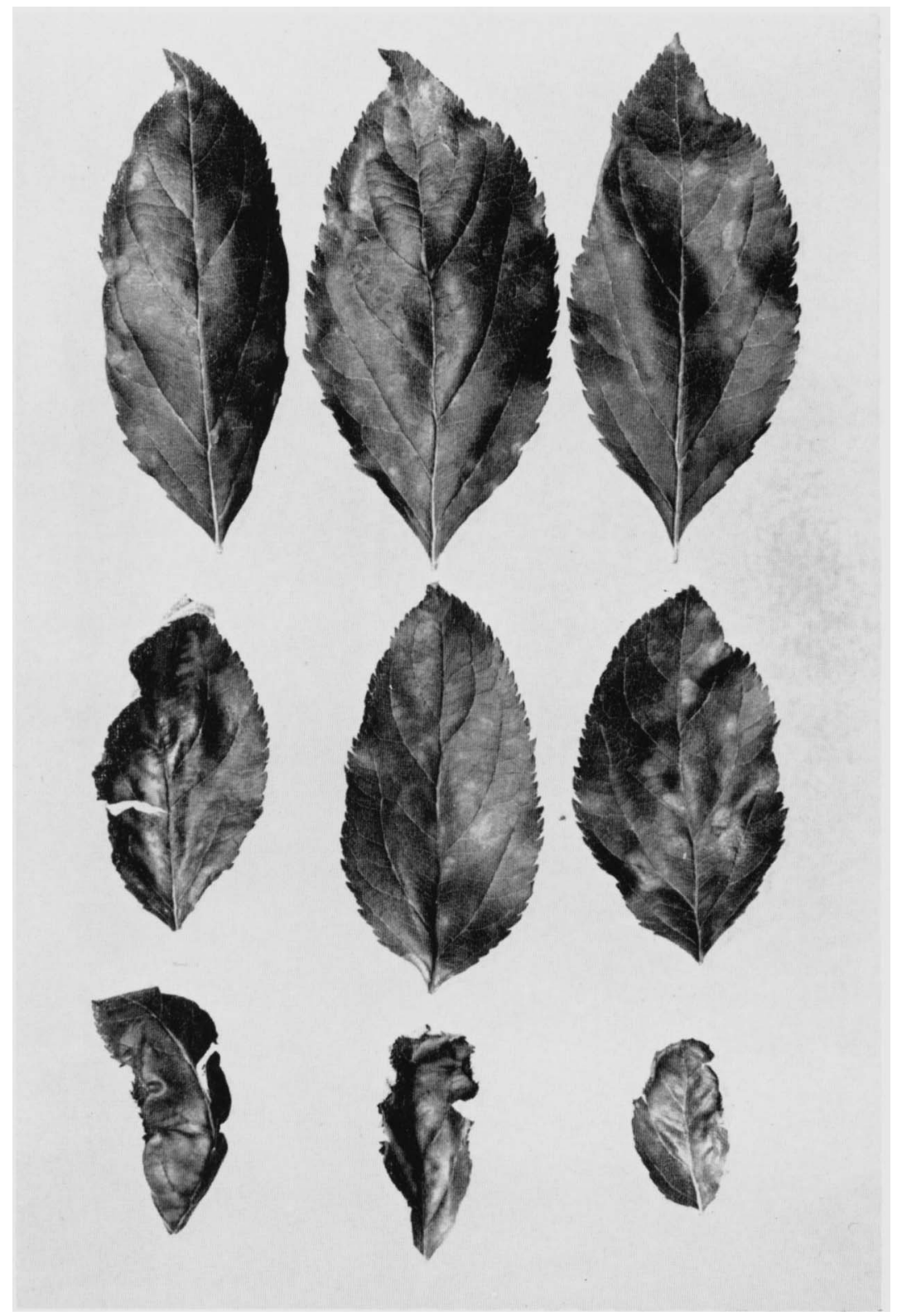

D. S. Kinkham-Host kelations of Venturla spp. Plate 1 